\title{
NEURAL CONTROL OF HATCHING: FATE OF THE PATTERN GENERATOR FOR THE LEG MOVEMENTS OF HATCHING IN POST- HATCHING CHICKS ${ }^{1}$
}

\author{
ANNE BEKOFF ${ }^{2}$ AND JULIE A. KAUER ${ }^{3}$ \\ Behavioral Biology Group, Department of Environmental, Population, and Organismic Biology, University of Colorado, Boulder, \\ Colorado 80309
}

Received October 15, 1981; Revised May 17, 1984; Accepted May 18, 1984

\begin{abstract}
Chicks from 0 to 61 days post-hatching were gently folded into the hatching position and placed in artificial glass eggs. Within 0 to 2 min they began to produce a behavior that qualitatively resembled normal hatching. Furthermore, quantitative analysis of electromyographic records showed that under these conditions the intralimb and interlimb leg motor output patterns produced during each hatching episode (the episode motor program) were those typical of normal hatching. The only major change associated with increasing posthatching age was a gradual increase in inter-episode interval. Therefore, we conclude that the neural patterngenerating circuitry which produces the motor program for the leg movements of hatching remains functional in post-hatching chicks despite the fact that, under normal conditions, hatching behavior is never used again.
\end{abstract}

Behaviors that are appropriate at one stage of development may not be appropriate at other stages and, therefore, during ontogeny, particular behaviors must be turned on and off at specific times. This is reflected in the appearance of "new" behaviors and the disappearance of "old" behaviors from an animal's repertoire. A variety of neural mechanisms may be involved in these behavioral transitions. For example, one mechanism would be to construct neural circuitry immediately prior to the stage at which the behavior is first needed and to dismantle it as soon as the behavior has served its purpose. Alternatively, the circuit could be present but could be either inhibited or lacking the necessary excitatory stimulus at all stages other than the one during which the behavior is required. A third possibility is that the neural circuit could be present throughout several developmental stages and could be used in the generation of different behaviors at different stages.

Several studies of the appearance of new behaviors during ontogeny in both invertebrates and vertebrates have shown that the underlying neural circuits are often constructed some time before a particular behavior is normally expressed (e.g., Blest, 1960; Bentley and Hoy, 1970; Bekoff et al., 1975; Bekoff,

\footnotetext{
${ }^{1}$ This work has been supported by National Science Foundation Grants BNS 79-13826 and 79-05770, and a fellowship from the Alfred P. Sloan Foundation to A. B. We thank Mr. E. Lutter for fabricating the glass eggs for us, Mr. W. A. Lavender for writing the computer programs, and Ms. J. Cavanagh and Ms. M. Marcotte for typing the manuscript. Drs. M. Bekoff, M. A. Corner, R. C. Eaton, and P. S. G. Stein made many useful comments on an earlier draft of the manuscript.

${ }^{2}$ To whom correspondence should be addressed.

${ }^{3}$ Present address: Department of Pharmacology, Yale University School of Medicine, New Haven, CT 06510.
}

1976; Kammer and Rheuben, 1976; Truman, 1976; Bekoff and Trainer, 1979; Bekoff and Lau, 1980). In some cases the neural circuits appear to be inhibited at early stages (Bentley and Hoy, 1970; Truman, 1976); in others the necessary conditions for turning them on are not normally present at early stages (Bentley and Hoy, 1970). In still others, it appears that the same neural circuit may be used to produce different behaviors at earlier developmental stages (Bekoff et al., 1975; Bekoff, 1976, 1981).

Much less attention has been focused on the fate of neural pattern-generating circuits after a particular behavior disappears from an animal's repertoire. Evidence available from studies of eclosion in silkmoths suggests that the neural circuits involved may be dismantled or permanently altered after completion of this behavior (Taylor and Truman, 1974; Truman, 1978). In order to analyze the neural mechanisms involved in turning off particular behaviors in vertebrates, we have examined hatching in the chicken.

Hatching consists of episodes of movement lasting 1 to $3 \mathrm{sec}$ that are repeated every 10 to $30 \mathrm{sec}$ and result in the chick's escape from the shell after 45 to $90 \mathrm{~min}$ (Hamburger and Oppenheim, 1967; Kovach, 1970; Oppenheim, 1973; Bakhuis, 1974; Bekoff, 1976). Each hatching episode consists of a highly stereotyped sequence of movements including rotation of the upper body, back thrusts of the head during which the beak hits against the shell, and strong thrusting movements of the legs which appear to provide the force for the body rotation (Hamburger and Oppenheim, 1967; Bakhuis, 1974). In the present study, we have concentrated on the leg movements of hatching because they are a prominent and distinctive part of the hatching behavior and because previous studies have provided precise, quantitative information on leg motor output in chick embryos (Bekoff et al., 1975; Bekoff, 1976).

The pattern-generating circuitry for the leg movements of 
hatching in chickens develops very early in embryonic life and then is gradually refined during the incubation period (Bekoff et al., 1975; Bekoff, 1976). However, under normal circumstances the hatching behavior itself occurs only once, at the end of the incubation period (Hamburger and Oppenheim, 1967; Kovach,1970). Therefore, it is of interest to determine the fate of the pattern-generating circuitry for the leg movements of hatching after normal hatching is completed.

Several previous studies have shown that the timing mechanism responsible for turning on movement episodes in the regular pattern typical of hatching is still present in posthatching chicks (Corner and Bakhuis, 1969; Corner et al., 1973; Bakhuis and van de Nes, 1979). These studies have shown that this "episode timer" can be activated by placing post-hatching chicks in the hatching position in glass eggs or by restraining chicks in any one of a variety of other ways.

Previous studies have not analyzed in detail the motor output patterns involved in the motor program used by post-hatching chicks during each leg movement episode in glass eggs to determine whether they represent the output of the patterngenerating circuitry which is used during normal hatching in the 20- to 21-day-old chick embryo. Because of the small amplitude of the leg movements within the egg, we have found that quantitative analysis of electromyographic (EMG) records permits more reliable and detailed characterization of the motor output pattern than does behavioral observation alone. Furthermore, we have found that the "episode timer" can regulate the timing of motor output patterns that are quite distinct from those seen during normal hatching (Bekoff and Kauer, 1982) and thus appears to represent a separate level of control of the behavior, perhaps analogous to the "bout timer," found by Carlson (1977) to be involved in turning on different motor programs during ecdysis in crickets.

In the present study, we have analyzed the pattcrns of intralimb and interlimb coordination to determine whether the pattern generating circuitry for the leg movements of hatching (as distinct from the "episode timer" that activates this circuitry) remains functional after completion of normal hatching or, alternatively, whether this circuitry is permanently altered, or even dismantled.

A preliminary account of some of these results has appeared (Bekoff and Kauer, 1980).

\section{Materials and Methods}

\section{Animals}

Fertile eggs from Grey Leghorn and Shaver strain chickens were obtained from Great Western Poultry, Inc. (Greeley, CO) and were incubated under standard conditions. Chicks typically hatched on day 21 of incubation and the day of hatching was called post-hatching day 0 . After hatching, chicks were maintained in groups in heated chambers with food and water ad libitum. No differences were found in the results obtained from the two strains of chickens; therefore, they have been combined.

\section{Experimental conditions}

Normal hatching. Eggs of 21-day-old chick embryos which had pipped or had begun to rotate were placed horizontally in a heated, humidified chamber (Bekoff, 1976). In order to allow EMG recordings and behavioral observations of the leg motor output produced during normal hatching, a hole was chipped through the shell over one or both legs. As soon as the blood supply to the chorioallantoic membrane was clamped off, the membrane was removed to allow better visibility of the legs. Hatching episodes appeared spontaneously.

Glass egg experiments. To determine whether the leg motor output typical of hatching could be elicited again in post-hatching chicks, 0to 61-day-old chicks were gently folded into the hatching position with the head bent to the right and tucked underneath the right wing (Hamburger and Oppenheim, 1967). Each chick was then placed into an artificial glass egg (Fig. 1; Bakhuis, 1974) of the appropriate size (i.e., close fitting but not so small as to restrict breathing). The two halves of the egg were taped together with Scotch tape. Each egg had holes for ventilation and for the leads of EMG recording electrodes. The egg was then placed horizontally on a plastic egg holder. Temperature was maintained at about $34^{\circ} \mathrm{C}$ with a lamp.

\section{Data collection}

Behavioral observations were made on chicks of all ages. In addition, both EMG and videotape recordings were made from chick embryos during normal hatching and from 0 - to 50-day-old post-hatching chicks in glass eggs.

$E M G$ recordings. EMG recordings were made from the following muscles: the hip extensor, semitendinosus (ST; also called caudilioflexorius; terminology of Landmesser (1978) and Hollyday (1980)); the hip flexor, sarturius (SA); the knee extensor, femorotibialis (FT; also called quadriceps femoris; terminology of Bekoff (1976)); the knee flexor, biceps femoris (BF; also called iliofibularis; terminology of Landmesser (1978) and Hollyday (1980)); the ankle extensor, gastrocnemius pars externa (GL; also called gastrocnemius lateralis; terminology of Bekoff (1976) and Landmesser (1978)); and the ankle flexor, tibialis anterior (TA). Muscle terminology is taken from Cracraft (1971) (see also Hudson et al., 1959; George and Berger, 1966). Functions were assigned to the six muscles based on their anatomical connections and whether they were active during extension or flexion of the leg during normal hatching. These muscles were chosen because they each showed clear activity either during leg extension or flexion, but not both. In contrast to Bekoff (1976) we have found that ST is often active exclusively during hip extension. It may be that this muscle is subdivided into a region active only during hip extension and a region active during both hip extension and knee flexion. However, this was not systematically explored in this study, nor have recordings been made from the accessory ST.

For analyses of intralimb coordination, all recordings were made from muscles of the right leg. For analyses of interlimb coordination recordings were made from GL and TA of both legs. In the figures, $r=$ right leg and $l=$ left leg.

During normal hatching, recordings were made from selected leg muscles using suction electrodes (Bekoff, 1976) or bipolar hook electrodes made from Teflon-coated stainless steel wires $(100 \mu \mathrm{m}$ diameter). A small incision was made in the skin overlying each muscle from which recordings were to be made. Suction electrodes were positioned on the surface of the muscles of interest; wire electrodes were inserted under visual control using a 25 gauge syringe needle. Wirc clectrodes were led directly to brass spring clamp connectors (Basmajian et al., 1966). A ground wire was placed within the egg.

To make recordings from chicks in glass eggs, post-hatching chicks were anesthetized with ketamine $(250 \mathrm{mg} / \mathrm{kg})$ or halothane and selected muscles were implanted with wire electrodes as described above. In the post-hatching chicks, the wires were led up underneath the skin to the midlumbar region and there were attached to long flexible leads. A ground wire was placed under the skin of the back. All incisions were closed with cyanoacrylate adhesive. Recordings were made after the chicks had completely recovered from the anesthesia (about $6 \mathrm{hr}$ for ketamine and $1 \mathrm{hr}$ for halothane). Intervals between leg movement

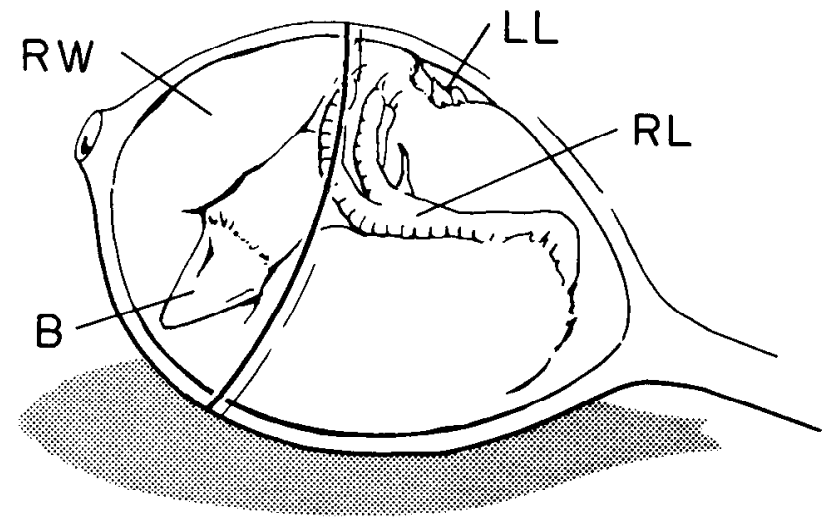

Figure 1. A 1-day-old post-hatching chick folded into the hatching position inside a glass egg. $B$, beak; $R W$, right wing; $L L$, left leg; $R L$, right leg. 
episodes were timed with a digital stopwatch. Records were taped on a TEAC 3340 s tape recorder for later filming and analysis. At the end of each experiment, the precise location of each electrode was determined by dissection.

Videotape recordings. A Sony videocamera and recorder were used to record behavior of the chicks in glass eggs and during normal hatching to allow qualitative comparisons to be made.

\section{Data analysis}

Parameters measured directly from the EMG records and digitized were: muscle burst duration, interburst interval, cycle duration, and episode duration (for definitions, see Fig. 2f). In addition, mean interepisode interval (mean number of seconds from the onset of one leg movement episode to the onset of the next episode) was calculated using data from a digital stopwatch.

To analyze intralimb coordination, phase relationships of various

TABLE I

Temporal characteristics of EMG records

\begin{tabular}{lcccc}
\hline \multirow{2}{*}{$\begin{array}{l}\text { Parameter Meas- } \\
\text { ured }\end{array}$} & $\begin{array}{c}\text { Normal Hatching } \\
\text { 20- to 21-Day } \\
\text { Embryos }\end{array}$ & \multicolumn{3}{c}{ Glass Egg Hatching Chicks } \\
\cline { 3 - 5 } & $(208)^{a}$ & $(200)$ & $(135)$ & $(30)$ \\
Inter-episode & $\left(8.6 \pm 7.7^{b}\right.$ & $20.8 \pm 7.4$ & $32.2 \pm 10.2$ & $55.1 \pm 24.3$ \\
Interval & $(94)$ & $(94)$ & $(65)$ & $(38)$ \\
Episode & $1.5 \pm 0.3$ & $1.3 \pm 0.5$ & $1.4 \pm 0.3$ & $1.8 \pm 0.7$ \\
Duration & $(103)$ & $(100)$ & $(82)$ & $(36)$ \\
Period & $1.6 \pm 0.2$ & $1.3 \pm 0.3$ & $1.1 \pm 0.4$ & $1.3 \pm 0.2$ \\
Gastrocnemius & $(103)$ & $(100)$ & $(82)$ & $(36)$ \\
Duration & $0.8 \pm 0.1$ & $0.7 \pm 0.3$ & $0.8 \pm 0.2$ & $0.8 \pm 0.3$ \\
Gastrocnemius & &
\end{tabular}

${ }^{a}$ Numbers in parentheses, number of embryos or chicks.

${ }^{b}$ Values are mean $\pm \mathrm{SD}$, in seconds.

\section{a.}

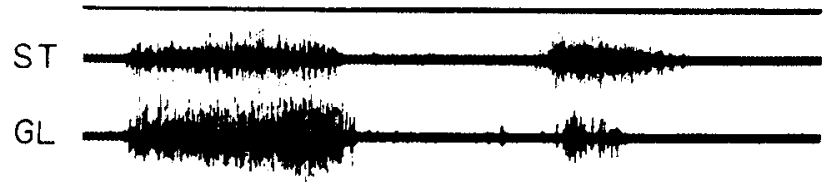

b.

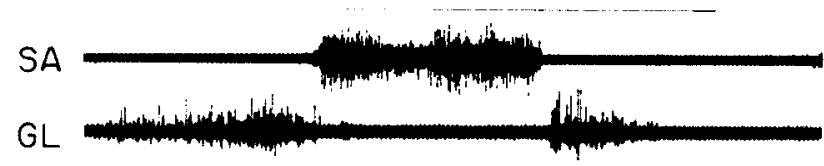

C.

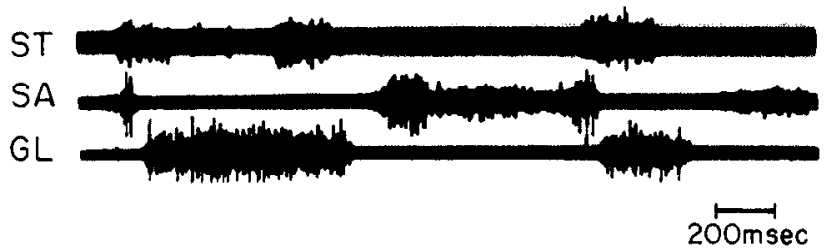

right leg muscles with respect to right gastrocnemius were calculated from EMG records. To analyze interlimb coordination, phase relationships of left gastrocnemius with respect to right gastrocnemius were calculated from EMG records.

\section{Results}

Behavioral observations. When 0- to 61-day-old post-hatching chicks were gently folded into the hatching position and placed in a close fitting glass egg (Fig. 1), they rapidly ceased struggling and vocalizing. Then, typically within 0 to 2 min, highly periodic episodes of movements began. These were accompanied by distinctive loud, regular peeps which sounded similar to the vocalizations heard during normal hatching. The movements appeared quite stereotyped. The most obvious components were rotary movements of the upper body accompanied by back thrusts of the head and strong, synchronous thrusting movements of the legs. These movements resulted in rotation of the chick as is seen during normal hatching although, of course, the beak could not crack the glass egg shell. More precise quantitative analyses of the leg movements involved in this behavior were carried out in 0 - to 15 -day-old post-hatching chicks. In the following sections these analyses are described and are compared with analyses of normal hatching.

Some chicks of all ages remained totally inactive when placed in the hatching position in a glass egg. However, a chick that did not respond in one trial usually did in a later trial. There did not appear to be a consistent circadian rhythm in responsiveness.

Temporal characteristics. To compare some of the temporal characteristics of leg movement episodes during glass egg behavior and normal hatching, mean inter-episode interval, epi-

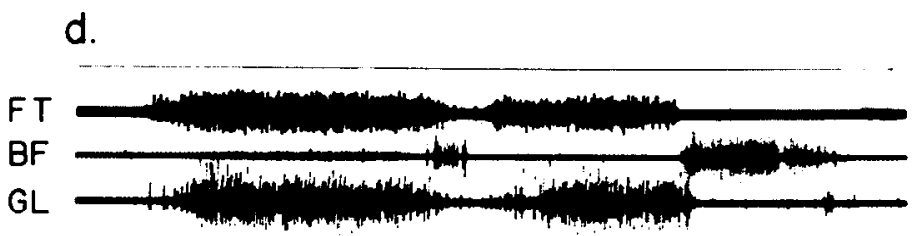

\section{e.}

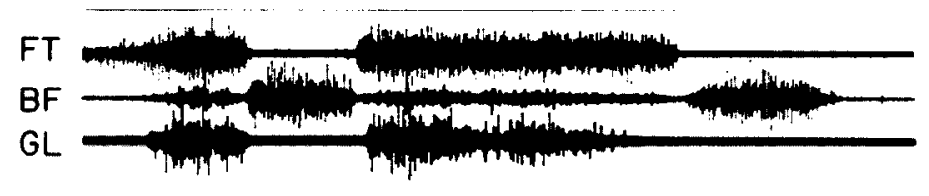

f.

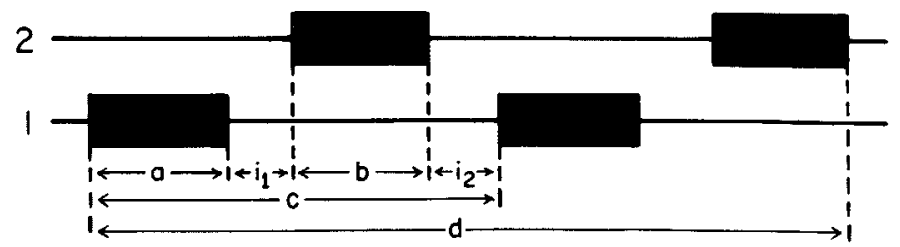

Figure 2. Intralimb motor output patterns: EMG records from right leg muscles showing consistent, easily recognizable patterns of muscle activation organized into short, one- to two-cycle episodes. $a$ and $b$, Normal hatching, hip $(S T, S A)$ and ankle $(G L)$ muscles. $c$, Glass egg behavior, hip $(S T, S A)$ and ankle $(G L)$ muscles. $d$, Normal hatching, knee $(F T, B F)$ and ankle $(G L)$ muscles. $e$, Glass egg behavior, knee ( $F T$, $B F$ ) and ankle $(G L)$ muscles. $f$, Parameters used in analyzing EMG records (after Reingold and Camhi, 1977): $a$, muscle 1 burst duration; $b$, muscle 2 burst duration; $c$, cycle duration; $d$, episode duration; $i_{1}$, interburst interval between muscle 1 and muscle 2 , defined as negative if bursts overlap; $i_{2}$, interburst interval between muscle 2 and muscle 1 . Phase of muscle 2 with respect to muscle 1 is defined as $\left(a+i_{1}\right) / c$. Gastrocnemius was always used as the reference trace, muscle 1 . Up to four channels per record were analyzed. 
NORMAL HATCHING

GLASS EGG HATCHING
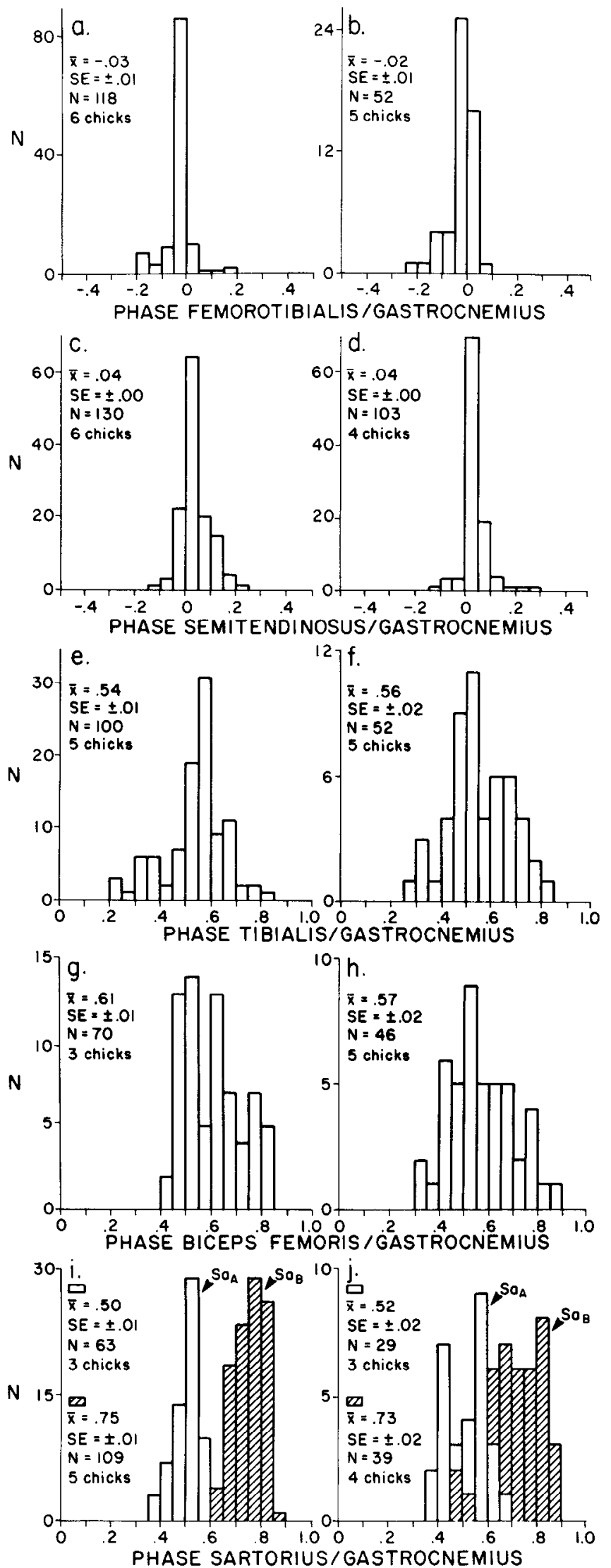

Figure 3. Intralimb motor output patterns: Histograms of the phase relationships of hip, knee, and ankle muscle activity with respect to gastrocnemius (ankle extensor) activity in normal hatching in 20- to sode duration, period length, and gastrocnemius burst duration were calculated (Table I).

One-way analyses of variance were carried out to determine whether there were statistically significant $(p<0.05)$ differences among the means. The Scheffe a posteriori test was used to determine where the significant differences occurred. These analyses revealed that the inter-episode intervals for normal hatching and glass egg behavior in 0 - to 2-day-old post-hatching chicks were not significantly different. However, mean interepisode intervals for glass egg behavior in 5- to 7-day-old chicks and 13- to 14-day-old chicks were significantly different from each other and from the younger chicks. The trend was toward increasing inter-episode interval with increasing age.

Although significant differences were found among the means for episode duration, they showed no consistent trend. The statistical analyses showed no significant differences between normal hatching and glass egg behavior in 5- to 7-dayold chicks, or between glass egg behavior in 0- to 2-day-old and 5 - to 7-day-old chicks. However, significant differences were found between normal hatching and glass egg behavior in 0 - to 2-day-old and 5- to 7-day-old chicks. Episode duration in 13to 14-day-old chicks was significantly longer than in any of the groups of younger chicks. Furthermore, although there was some inter-individual variation, the number of extension-flexion sequences per episode stayed relatively constant. That is, at all ages, in both normal hatching and glass egg behavior, approximately 30 to $40 \%$ of the episodes consisted of one sequence and 50 to $60 \%$ consisted of two sequences. Relatively few ( 0 to $10 \%$ ) three-sequence episodes were observed.

Gastrocnemius period also showed no consistent trend. Mean values for glass egg behavior in 0 - to 2-day-old and 13- to 14day-old post-hatching chicks did not differ significantly. However, mean values for normal hatching and glass egg behavior in 5- to 7-day-old chicks differed both from each other and from the other two groups.

No significant differences ( $p>0.15$ ) were found among the mean values for gastrocnemius burst duration.

Intralimb coordination. EMG recordings from ankle, knee, and hip muscles of the right leg showed that, during normal hatching episodes in 21-day-old embryos, all three joints extended together and the intralimb muscle activity pattern was one of altenation of extension and flexion, usually organized into one to three extension-flexion sequences (Fig. 2, $a, b$, and $d$; see also Bekoff, 1976). Extension almost always began the sequence. EMG recordings from 0 - to 1 -day-old post-hatching chicks during leg movement episodes in a glass egg (Fig. 2, $c$ and $e$ ) showed the same pattern. Phase histograms (Fig. 3) showed striking similarities in intralimb muscle coordination between normal hatching and glass egg behavior. During normal hatching, the knee extensor, femorotibialis, and the hip extensor, semitendinosus, were both activated near phase 0.0 with respect to the ankle extensor, gastrocnemius ( $F i g .3, a$ and c). That is, these hip, knee, and ankle extensors were activated synchronously. The ankle flexor, tibialis, and the knee flexor,

21-day-old embryos and in glass egg behavior in 0- to 1-day-old posthatching chicks. $a$, Normal hatching, knee extensor; $b$, glass egg behavior, knee extensor. In both $a$ and $b$ femorotibialis is coactivated with gastrocnemius. $c$, Normal hatching, hip extensor; $d$, glass egg behavior, hip extensor. In both $c$ and $d$ semitendinosus is coactivated with gastrocnemius. $e$, Normal hatching, ankle flexor; $f$, glass egg behavior, ankle flexor. In both $e$ and $f$ tibialis is activated in alternation with gastrocnemius. g, Normal hatching, knee flexor; $h$, glass egg behavior, knee flexor. In both $g$ and $h$ biceps femoris is activated in alternation with gastrocnemius. $i$, Normal hatching, hip flexor; $j$, glass egg hatching, hip flexor. In both $i$ and $j$ sartorius is activated in alternation with gastrocnemius. In addition, it shows a double bursting pattern in both. The two bursts are labeled $S a_{A}$ and $S a_{B}$. 
biceps femoris, were activated with mean phase values between 0.5 and 0.7 (Fig. 3, $e$ and $g$ ). Thus, they were activated in alternation with the extensors. Sartorius was also activated in alternation with the extensors, but it showed a more complex, double bursting pattern (Figs. $2 b$ and $3 i$ ). During leg movement episodes recorded from 0- to 1-day-old post-hatching chicks in glass eggs, coactivation of extensors (Fig. $3, b$ and $d$ ) and alternation of flexors and extensors (Fig. $3, f, h$, and $j$ ) were also seen. In addition, the distinctive double bursting paltern of sartorius was present (Figs. $2 c$ and $3 j$ ). The Student's $t$ test (two-tailed) was used to compare mean phase values for each pair of muscles during normal hatching and glass egg behavior. No significant differences were found for any pair of muscles.

Results obtained from 6- to 7-day-old and 13- to 14-day-old post-hatching chicks were similar to those seen in the 0 - to 1day-old chicks. For example, phase FT,GL in a 6-day-old chick was $-0.01 \pm 0.04(N=24)$. In another 6-day-old chick, phase $\mathrm{SA}_{\mathrm{A}}, \mathrm{GL}$ was $0.59 \pm 0.11(N=11)$ and phase $\mathrm{SA}_{\mathrm{B}}, \mathrm{GL}$ was 0.75 $\pm 0.12(N=5)$. For two 13 -day-old chicks phase $\mathrm{SA}_{\mathrm{A}}, \mathrm{GL}$ was $0.58 \pm 0.13(N=9)$ and phase $\mathrm{SA}_{\mathrm{B}}, \mathrm{GL}$ was $0.71 \pm 0.11(N=$ 9). For three 13- to 14-day-old chicks phase TA,GL was $0.61 \pm$ $0.15(N=32)$.

One-way analysis of variance followed by the Scheffe test revealed no statistically significant differences between any of these phase values and the values found for 0 - to 1-day old post-hatching chicks. Thus, no age-related trends were found among the post-hatching chicks. Furthermore, the phase values for FT,GL and $\mathrm{SA}_{\mathrm{B}}$, GL for glass egg behavior in 6-day-old and/ or 13- to 14-day-old post-hatching chicks did not significantly differ from the phase values for normal hatching. In two cases, phase TA,GL and phase $\mathrm{SA}_{\mathrm{A}}, \mathrm{GL}$, the values for glass egg behavior at 6 days and/or 13 to 14 days post-hatching were significantly different from the values for normal hatching, although they were not significantly different from one another or from the values for 0- to 1-day-old chicks.

Interlimb coordination. To characterize further the leg motor output of post-hatching chicks in glass eggs, the pattern of

a.

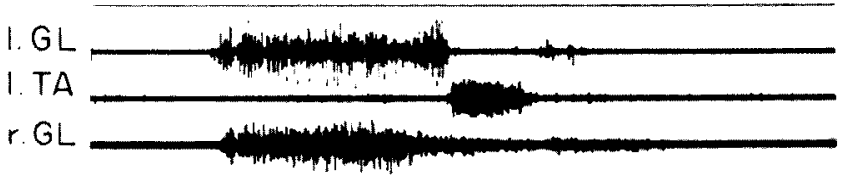

b.

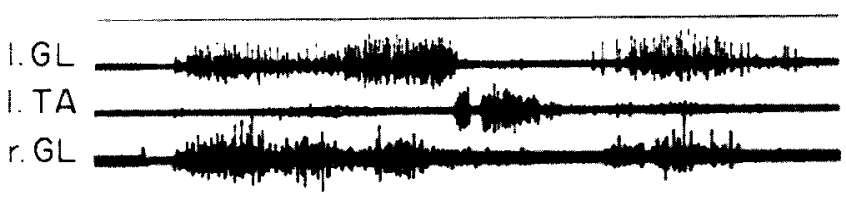

C.

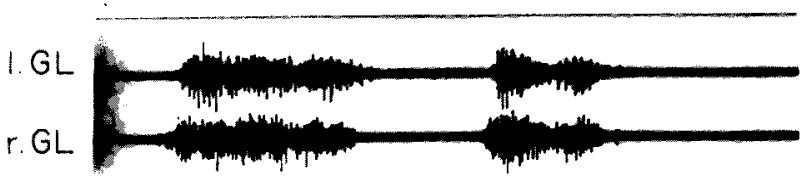

interlimb coordination was analyzed and compared with that seen during normal hatching. Figure $4, a$ and $b$, shows typical examples of EMG recordings from right and left leg ankle muscles during normal hatching. The ankle extensors of the two legs were coactivated as were the ankle flexors. Similar EMG recordings taken from 1-day-old post-hatching chicks in glass eggs are shown in Figure $4, c$ and $d$. Figure $4 e$ shows an EMG record from a 14-day-old and Figure $4 f$ shows an EMG record from a 50-day-old post-hatching chick. Phase histograms constructed from data from normal embryonic hatching (Fig. 5 a) and glass egg behavior in 1-day-, 6-day-, and 13- to 15-dayold post-hatching chicks (Fig. $5, b$ to $d$ ) show mean phase values of 0.0 , indicating that a synchronous pattern of interlimb coordination was consistently present in all cases.

Comparisons with other behaviors. To determine whether the leg motor output of normal hatching and glass egg behavior differ substantially from other behaviors involving leg movements, walking and hopping were analyzed. Walking was chosen because it is the most common bchavior involving leg movements performed by post-hatching chicks. Hopping was chosen because, like hatching, it involves a synchronous interlimb coordination pattern.

Both walking and hopping motor output patterns differed from hatching in that they did not occur in a regular episodic pattern. Furthermore, the interlimb pattern of coordination seen during walking was one of alternation (Fig. 6, $a$ and $b$ ). The interlimb coordination pattern during hopping, however, was one of synchrony (Fig. 6, $c$ and $d$ ). A Student's $t$ test showed that the interlimb phase values for normal hatching (Fig. $5 a$ ) and hopping (phase l.GL,r.GL $=0.02 \pm 0.15 ; N=$ 118) were not significantly different.

Intralimb patterns of coordination for walking and hopping differed from those of hatching in several ways. For example, in both hopping and walking, sartorius showed only a single burst in each gastrocnemius period (Fig. 6, $a$ and $c$ ). Femorotibialis showed a distinctive double bursting pattern during walking (Fig. $6 a$ ) and, although only a single burst occurred

d.

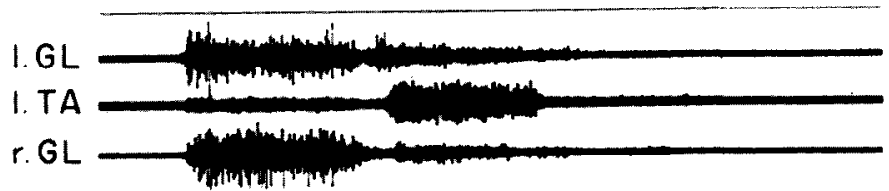

e.

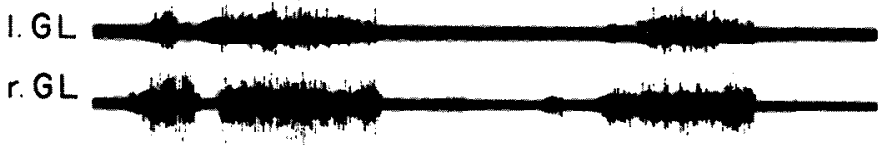

f.

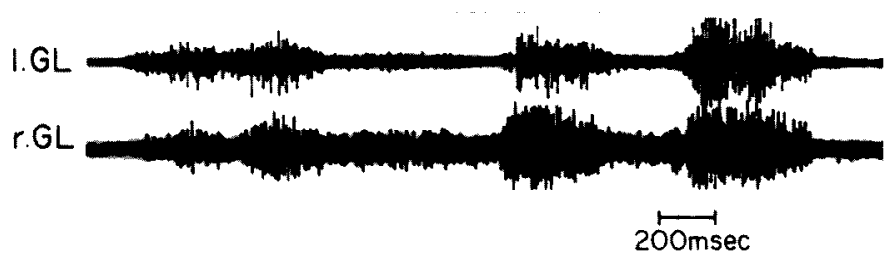

Figure 4. Interlimb motor output patterns: EMG recordings from the right and left leg ankle extensor muscles $(r$. and $l$. GL) and an ankle flexor (TA). $a$ and $b$, Normal hatching, 21-day-old embryos. $c$ and $d$, Glass egg behavior, 1-day-old post-hatching chicks. $e$, Glass egg behavior, 14-day-old post-hatching chick. $f$, Glass egg behavior, 50-day-old post-hatching chick. 

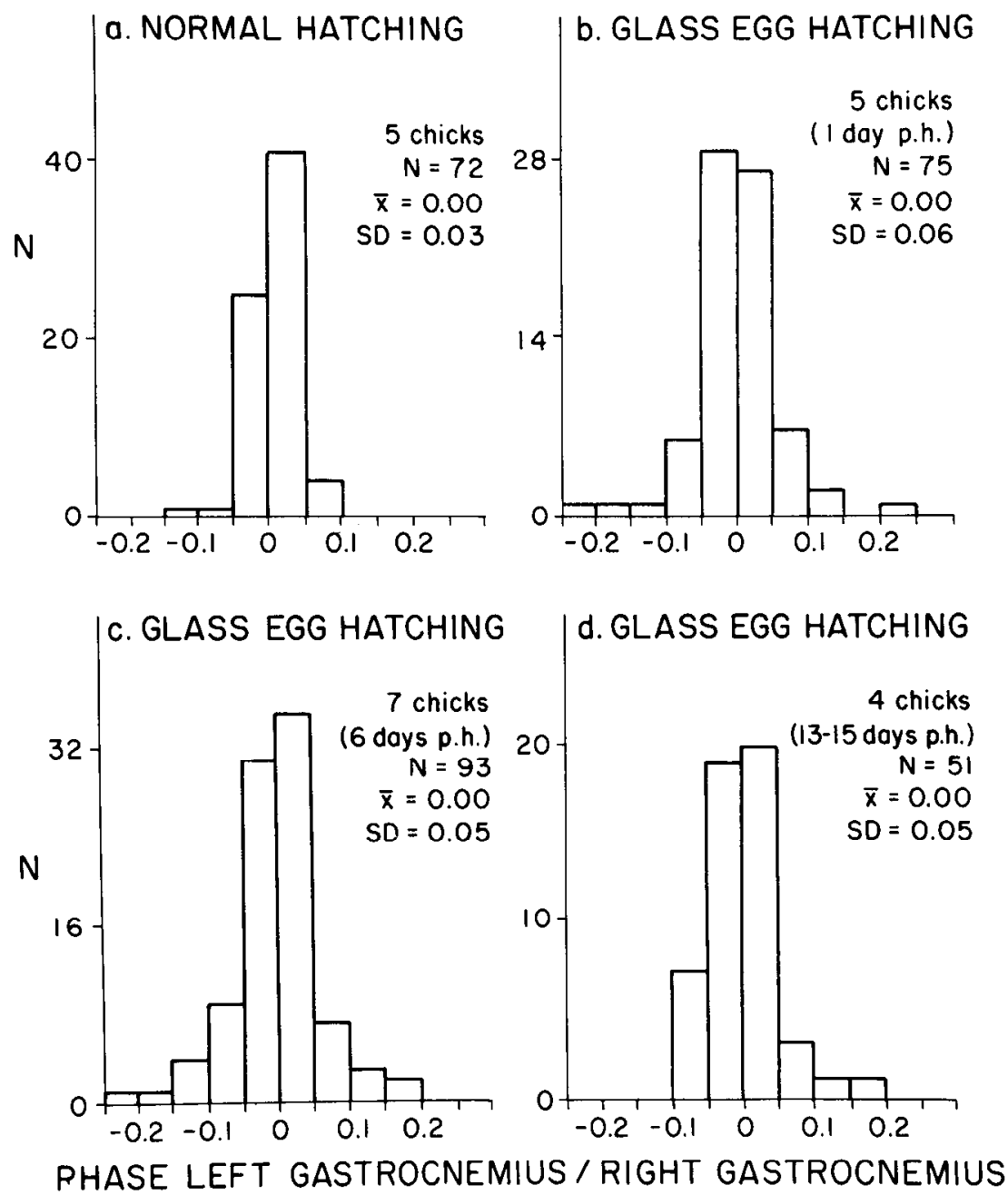

Figure 5. Interlimb motor output patterns: Histograms of the phase relationship of left gastrocnemius with respect to right gastrocnemius. $a$, Normal hatching, 21-day-old embryos. $b$, Glass egg behavior, 1-day-old post-hatching chicks. $c$, Glass egg behavior, 5- to 7-day-old post-hatching chicks. $d$, Glass egg behavior, 13- to 15-day-old posthatching chicks. In all three cases mean phase values are 0.0 , indicating a pattern of synchronous activation of right and left gastrocnemius muscles.

during hopping (Fig. 6d), the mean phase value (phase FT,GL $=0.15 \pm 0.11 ; N=78$ ) was significantly different from that seen during hatching (Fig. $3, g$ and $h$ ). For some other muscles the pattern was not strikingly different. For example, semitendinosus was activated with a mean phase value near 0.00 in all three behaviors. A more detailed analysis of the similarities and differences among these and other leg motor output patterns in the chick is currently in preparation (A. Bekoff, unpublished data).

\section{Discussion}

Based on the temporal characteristics of the leg movement episodes (Table I), rotation in the egg, and vocalizations, it is clear that placing a post-hatching chick in the hatching position in an artificial egg elicits a behavior that resembles normal hatching in several respects. These results confirm the observations of Corner and colleagues (Corner and Bakhuis, 1969; Corner et al.,1973; Bakhuis and van de Nes, 1979) and suggest that the "episode timer" for hatching does remain functional after hatching. However, we did find a gradual, age-related increase in inter-episode interval.

Because the episode timing mechanism and the patterngenerating mechanism for the leg movement motor program appear to be separable units of the motor control circuitry for hatching (Bekoff and Kauer, 1982), we focused next on an analysis of leg motor output to determine the fate of the pattern generator for the leg movements of hatching in the posthatching chick.

As initially shown by Bekoff (1976) and extended to additional muscles in the present study, the intralimb pattern of leg motor output during normal hatching consists of one to three extension-flexion sequences during which hip, knee, and ankle extensors are coactivated in alternation with the hip, knee, and ankle flexors (Figs. 2 and 3 ). In addition, the characteristic interlimb pattern of coordination is one of synchronous activation of right and left legs (Figs. 4 and 5). This result is in contrast to several reports of alternating leg movements during hatching (Hamburger and Oppenheim, 1967; Kovach, 1970). However, our results do agree with behavioral data presented by Bakhuis (1974), based on extensive analysis of films of hatching embryos, except that Bakhuis (1974) typically observed only one extension-flexion sequence per episode. This finding emphasizes the need for the kind of precise and detailed data that can be obtained from either EMG records or frameby-frame film (or videotape) analysis when analyzing behaviors involving relatively small amplitude movements (Bekoff, 1978). 

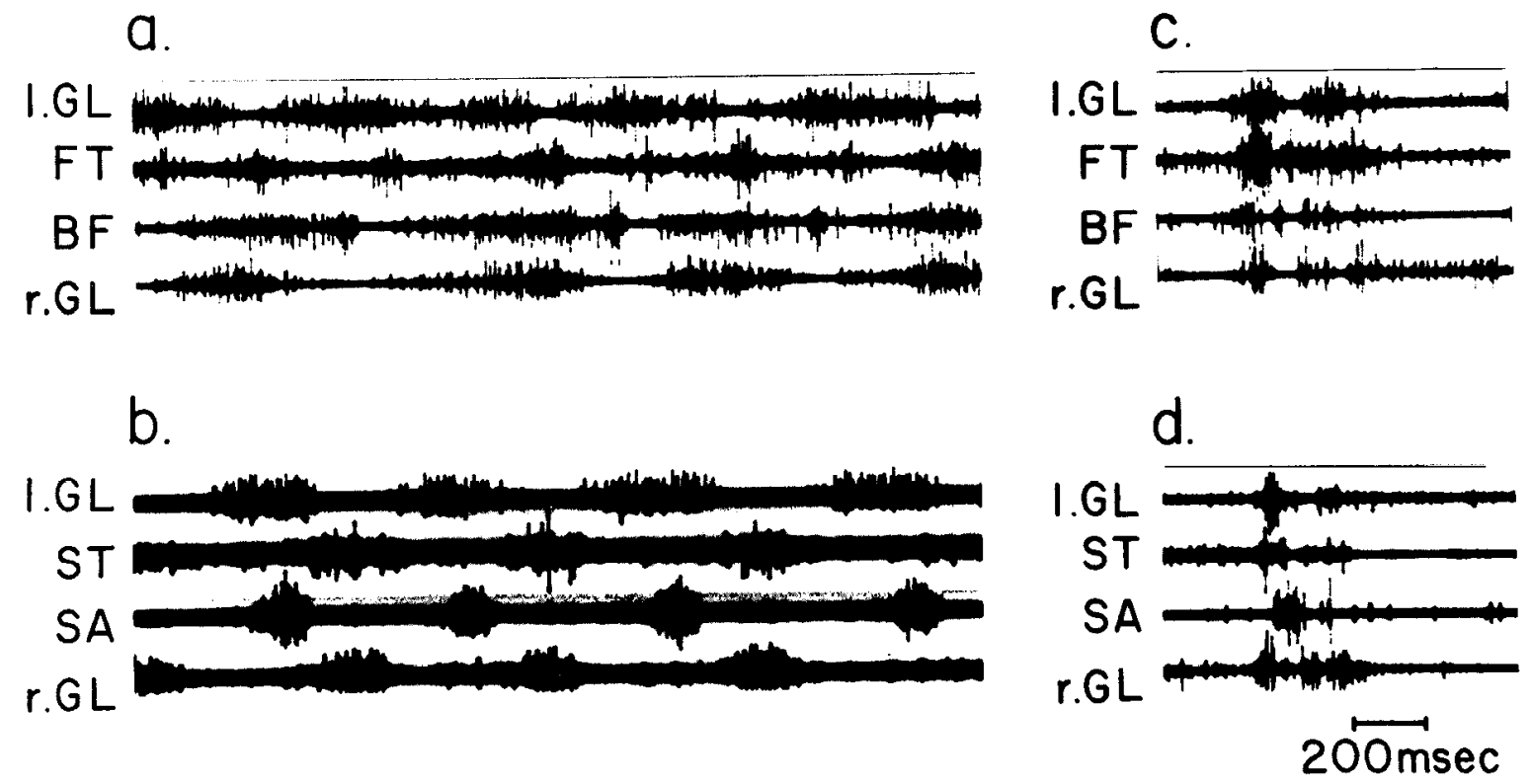

Figure 6. Walking and hopping: EMG records from hip, knee, and ankle muscles. $a$, Walking, right and left leg ankle extensor muscles (GL) and right leg knee extensor $(F T)$ and flexor $(B F)$. b, Walking, right and left leg ankle extensors and right leg hip extensor (ST) and flexor (SA). $c$, Hopping, right and left leg ankle extensors and right leg knee extensor and flexor. $d$, Hopping, right and left leg ankle extensors and right leg hip extensor and flexor.

Our results show that both the intralimb and interlimb patterns of motor output which have been found to be typical of hatching are produced by the post-hatching chick when it is placed in the hatching position in a glass egg. Although the hatching motor output cannot be elicited as reliably at older stages, when it does appear, the intralimb and interlimb coordination patterns are normal. Furthermore, new or distinctly different patterns do not appear. Thus, the only major change associated with increasing age is a gradual increase in interepisode interval; the basic leg motor output pattern within an episode does not change.

Therefore, we conclude that, in addition to the "episode timer," the neural pattern-generating circuitry that produces the motor program for the leg movements of hatching also remains functional in the post-hatching chick despite the fact that, under normal circumstances, hatching behavior is never used again. Furthermore, this pattern-generating circuit can be turned on by placing the chick in a glass egg at least up to $81 / 2$ weeks post-hatching, by which time the chicken is essentially a young adult in terms of motor development (Kruijt, 1964). Bekoff and Kauer (1982) have shown that bending the neck to the right or to the left is the necessary and sufficient condition for turning on the hatching leg motor output in 0-day-old posthatching chicks. The results presented here rule out the possibility that the pattern-generating circuit for the leg movements of hatching is dismantled or permanently modified even long after hatching has been completed.

We have also presented evidence showing that the leg motor output of hatching is distinctive. It can be easily distinguished from walking motor output by numerous parameters, including intra- and interlimb coordination patterns and the absence of regular periodic episodes (see also Jacobson and Hollyday, 1982). In addition, when hatching is compared to hopping, a post-hatching locomotory behavior which, like hatching, involves synchronous interlimb coordination, substantial differences are found in intralimb coordination, and periodic episodes are again absent. It is clear, then, that the leg motor output pattern produced by a post-hatching chick placed in a glass egg is that of hatching and not one of the other behaviors commonly produced by post-hatching chicks.
This is not to say that the same, or elements of the same, neural circuitry that is used to produce the leg movements of hatching may not be used later in locomotory behaviors. The question of what relationship exists between the pattern-generating circuit for the leg movements of hatching and patterngenerating circuits used to produce other motor behaviors in the post-hatching chick remains to be answered. Because it is still present but has completed its role in hatching, one possibility is that this circuit (or elements of it) may be reused to produce walking (Bekoff, 1978) and other locomotory behaviors such as jumping and hopping. The leg motor output patterns involved in these behaviors each differ in several ways from those typical of hatching (Fig. 6; A. Bekoff, unpublished data). Thus, if the hatching pattern-generating circuit is reused to produce any of them, its output must be modified. The results of the present study make it clear that the hatching patterngenerating circuit is not permanently modified. Recent results support the hypothesis that short-term modulation by sensory input is involved (Bekoff, 1982).

\section{References}

Bakhuis, W. L. (1974) Observations on hatching movements in the chick (Gallus domesticus). J. Comp. Physiol. Psychol. 87: 997-1003.

Bakhuis, W. L., and J. C. M. van de Nes (1979) The causal organization of climax behavior in the domestic fowl (Gallus domesticus). Behaviour 70: 185-230.

Basmajian, J. V., W. J. Forrest, and G. Shine (1966) A simple connector for fine-wire EMG elestrodes. J. Appl. Physiol. 21: 1680.

Bekoff, A. (1976) Ontogeny of leg motor output in the chick embryo: A neural analysis. Brain Res. 106: 271-291.

Bekoff, $\Lambda$. (1978) $\Lambda$ neuroethological approach to the study of the ontogeny of coordinated behavior. In The Development of Behavior: Comparative and Evolutionary Aspects, G. M. Burghardt and M. Bekoff, eds., pp. 19-41, Garland Publishing, Inc., New York.

Bekoff, A. (1981) Embryonic development of the neural circuitry underlying motor coordination. In Studies in Developmental Neurobiology: Essays in Honor of Viktor Hamburger, W. M. Cowan, ed., pp. 134-170, Oxford University Press, New York.

Bekoff, A. (1982) Comparison of hatching and walking motor output patterns in normal and deafferented chicks. Soc. Neurosci. Abstr. 8: 738. 
Bekoff, A., and J. A. Kauer (1980) Neural control of hatching in the chick. Soc. Neurosci. Abstr. 6:75.

Bekoff, A., and J. A. Kauer (1982) Neural control of hatching: Role of neck position in turning on hatching leg movements in post-hatching chicks. J. Comp. Physiol. 145: 497-504.

Bekoff, A., and B. Lau (1980) Interlimb coordination in 20-day old rat fetuses. J. Exp. Zool. 214: 173-175.

Bekoff, A., and W. Trainer (1979) Development of interlimb coordination during swimming in postnatal rats. J. Exp. Biol. 83: 1-11.

Bekoff, A., P. S. G. Stein, and V. Hamburger (1975) Coordinated motor output in the hindlimb of the 7-day chick embryo. Proc. Natl. Acad. Sci. U. S. A. 72: 1245-1248.

Bentley, D. K., and R. R. Hoy (1970) Postembryonic development of adult motor patterns in crickets: A neural analysis. Science 70: 14091411.

Blest, A. D. (1960) The evolution, ontogeny and quantitative control of the settling movements of some New World saturniid moths, with some comments on distance communication by honey-bees. Behaviour 16: $188-253$.

Carlson, J. R. (1977) The imaginal ecdysis of the cricket (Teleogryllus oceanicus). I. Organization of motor programs and roles of central and sensory control. J. Comp. Physiol. 115: 299-317.

Corner, M. A., and W. L. Bakhuis (1969) Developmental patterns in the central nervous system of birds. V. Cerebral electrical activity, forebrain function and behavior in the chick at the time of hatching. Brain Res. 13: 541-555.

Corner, M. A., W. L. Bakhuis, and C. van Wingerden (1973) Sleep and wakefulness during early life in the domestic chicken and their relationship to hatching and embryonic motility. In Behavioral Embryology, G. Gottlieb, ed., pp. 245-279, Academic Press, Inc., New York.

Cracraft, J. (1971) The functional morphology of the hindlimb of the domestic pigeon, Columba livia. Bull. Am. Mus. Nat. Hist. 144: 171268.
George, J. C., and A. J. Berger (1966) Avian Myology, Academic Press, Inc., New York.

Hamburger, V., and R. Oppenheim (1967) Prehatching motility and hatching behavior in the chick. J. Exp. Zool. 166: 171-204.

Hollyday, M. (1980) Organization of motor pools in the chick lumbar lateral motor column. J. Comp. Neurol. 194: 143-170.

Hudson, G. E., P. J. Lanzillotti, and G. D. Edwards (1959) Muscles of the pelvic limb in galliform birds. Am. Midl. Nat. 61: 1-67.

Jacobson, R. D., and M. Hollyday (1982) A behavioral and electromyographic study of walking in the chick. J. Neurophysiol. 48: 238-256.

Kammer, A. E., and M. B. Rheuben (1976) Adult motor patterns produced by moth pupae during development. J. Exp. Biol. 65: 6584.

Kovach, J. K. (1970) Development and mechanisms of behavior in the chick embryo during the last five days of incubation. J. Comp. Physiol. Psychol. 73: 392-406.

Kruijt, J. P. (1964) Ontogeny of social behavior in Burmese red junglefowl (Gallus gallus spadiceus). Behavior (Suppl.) 12: 1-201.

Landmesser, L. T. (1978) The development of motor projection patterns in the chick hind limb. J. Physiol. (Lond.) 284: 391-414.

Oppenheim, R. (1973) Prehatching and hatching behavior: A comparative and physiological consideration. In Behavioral Embryology, G. Gottlieb ed., pp. 153-244, Academic Press, Inc., New York.

Reingold, S. C., and J. M. Camhi (1977) A quantitative analysis of rhythmic leg movements during three different behaviors in the cockroach, Periplaneta americana. J. Insect Physiol. 23: 1407-1420.

Taylor, H. M., and J. W. Truman (1974) Metamorphosis of the abdominal ganglia of the tobacco hornworm, Manduca sexta. J. Comp. Physiol. 90: 367-388.

Truman, J. W. (1976) Development and hormonal release of adult behavior patterns in silkmoths. J. Comp. Physiol. 107: 39-48.

Truman, J. W. (1978) Hormonal release of stereotyped motor programs from the isolated nervous system of the Cecropia silkmoth. J. Exp. Biol. 74: 151-173. 